\title{
Synthesis and characterization of atherosclerotic target anti-CD47 functionalized by nano-polyelectrolyte complexes between chitosan and hyaluronic acid and in vivo and in vitro targeting experiments
}

\author{
Jun Yu ${ }^{1, A}$, Qiurong Ruan ${ }^{2, E}, X_{i u ~ N i e}{ }^{1, E}$ \\ ${ }^{1}$ Department of Pathology, Union Hospital, Tongji Medicial College, Huangzhong University of Science and Technology, Wuhan, China \\ ${ }^{2}$ Institute of Pathology, Tongji Hosipital, Tongji Medical College, Huazhong University of Science and Techonlogy, Wuhan, China \\ A - research concept and design; $B$ - collection and/or assembly of data; $C$ - data analysis and interpretation; \\ $D$ - writing the article; $E$ - critical revision of the article; $F$ - final approval of the article
}

\section{Address for correspondence}

Jun Yu

E-mail:89679676@qq.com

\section{Funding sources}

This work was financially supported by the National Natural Science Foundation of China (grant No. 81200104), and the Youth Foundation of Huazhong University of Science and Technology (grant No. 02.03.2017-314).

Conflict of interest

None declared

Received on 0ctober 14, 2019

Reviewed on June 29, 2020

Accepted on September 20, 2020
Cite as

Yu J, Ruan Q, Nie X. Synthesis and characterization of atherosclerotic target anti-CD47 functionalized by nano-polyelectrolyte complexes between chitosan and hyaluronic acid and in vivo and in vitro targeting experiments. Adv Clin Exp Med. 2020;29(12):1407-1415. doi:10.17219/acem/127685

DOI

$10.17219 /$ acem/127685

Copyright

Copyright by Author(s)

This is an article distributed under the terms of the

Creative Commons Attribution 3.0 Unported (CC BY 3.0)

(https://creativecommons.org/licenses/by/3.0/)

\begin{abstract}
Background. In several different atherosclerotic model mice, blocking CD47 with anti-CD47 antibody significantly reduced accumulation of arterial plaque.

Objectives. We described the development of multifunctional positively charged polyelectrolyte complex (PEC) nanoparticles, designed to be stable at physiological salt concentrations and pH for effective targeted delivery in atherosclerosis.

Material and methods. These nanoparticles were obtained by charge neutralization using chitosan (CS) as the polycation and hyaluronic acid (HA) as the polyanion. An atherosclerotic-model antibody, the antiCD47 antibody, was sorbed onto the particle surfaces in water and phosphate-buffered saline (PBS) for $4 \mathrm{~h}$. The synthetic nanocarriers were exposed to vascular endothelial cells (VECS) in vitro to study their targeted adsorption to the cells, and the targeted distribution of nanocarriers was evaluated in vivo.

Results. We showed that the complexation process and the physicochemical properties of the resulting colloids were impacted by external parameters such as the charge mixing ratio and the polymer concentrations. Nonstoichiometric colloidal PECs were obtained in water or PBS (pH 7.4) and remained stable for 1 month. The morphology was studied with scanning electron microscopy (SEM). The average size of the CS-HA/ CD47 nanoparticles was 375-620 nm, with a positive zeta potential. The CD47-targeted nanocarriers could be efficiently adsorbed to the surface of VECs in vitro, and their targeted distribution was evaluated in vivo.

Conclusions. Targeted nanocarriers can be effectively adsorbed to the surface of a VEC line and atheromatous plaque in vitro and in vivo. These results demonstrated that CS-HA/CD47 can be an effective carrier for targeted drug delivery in atherosclerosis.
\end{abstract}

Key words: atherosclerosis, chitosan, hyaluronic acid, polyelectrolyte complexes, anti-CD47 


\section{Introduction}

In a recent study, researchers from the Stanford University School of Medicine (USA) found that cells with surface antigen CD47 are extremely abundant in atherosclerotic plaque, and that CD47-blocking antibodies reverse defects in efferocytosis, normalize the clearance of diseased vascular tissue and ameliorate atherosclerosis in multiple mouse models. In several different atherosclerotic mouse models, blocking CD47 with anti-CD47 antibody significantly reduced arterial plaque accumulation. ${ }^{1}$ However, how to use CD47 antibody to achieve effective drug delivery remains a key issue to be solved in future research.

In the past few years, the preparation of polyelectrolyte complexes (PECs) through the electrostatic interaction of oppositely charged polyions, especially as a drug carrier or gene delivery system, has been widely studied by researchers. $^{2,3}$ The preparation of PEC nanoparticles is relatively simple, and the synthesis requires minimal technical expertise and equipment. Without the need for toxic organic solvents and chemical cross-linking agents, PEC nanoparticles can be prepared under mild conditions and easily controlled.

There are many factors that influence the formation and physical properties of PEC nanoparticles, such as $\mathrm{pH}$, temperature, ionization, ionic strength, and interaction time. The stability of PECs depends on the relative molecular weight and charge mixing ratio. ${ }^{4}$ Chitosan (CS) is a polysaccharide obtained by partially deacetylated chitin. Chitosan has some properties that are particularly suitable for medical applications, such as biocompatibility, ${ }^{5}$ biodegradability and non-toxicity, ${ }^{6}$ in addition to being relatively inexpensive. ${ }^{7}$ The glucosamine residue of the primary amino group $\left(-\mathrm{NH}_{2}\right)$ can be protonated in a weakly acidic environment. Therefore, CS can be combined with various anionic forms of PECs such as dextran sulfate, ${ }^{8}$ chondroitin sulfate, ${ }^{9}$ sodium alginate-carboxymethylcellulose,$^{10-12}$ carrageenan, polygalacturonic acid, ${ }^{13,14}$ and DNA. Hyaluronic acid (HA) is a low-density, multi-component weak acid that is part of the extracellular matrix of all higher animals. It has high lubricity, water absorption and water retention, all of which affect the migration, adhesion and proliferation of various cells. ${ }^{15}$ Biomedical applications of HA include ophthalmic surgery, arthritis treatment, wound healing stents, tissue engineering, and implant materials. ${ }^{16-18}$ Hyaluronic acid can be used in biomedical applications with other polymers such as polylysine ${ }^{19,20}$ and silk fibroin to form PECs. Recent studies have shown that chitosan-hyaluronic acid PEC (CS-HA PEC) nanoparticles can be used as a vector for gene delivery, ${ }^{21-23}$ with vector efficiency of up to $60 \% .{ }^{24}$

Based on the above, the aim of this study was to combine the advantages of CS and HA to develop a polyelectrochemical composite PEC nanoparticle for CD47 antibody adsorption, and to study whether colloidal PEC nanoparticles are stable to the CD47 antibody. In vivo and in vitro cytology experiments with the synthetic anti-CD47-targeted nanocarriers were performed to evaluate the targeted adsorption of vascular endothelial cells (VECs) and atherosclerotic plaque.

This study was approved by the Ethics Committee of Tongji Medical College of Huazhong University of Science and Technology (Wuhan, China).

\section{Material and methods}

Chitosan with varying degrees of acetylation (DA) was provided by Jinan Haidebei Marine Biological Engineering Co., Ltd. (Jinan, China). The DA for the CS used were $5 \%$ and $48 \%$ and the molar molecular weight $(\mathrm{Mw})$ ranged from $100 \times 10^{3} \mathrm{~g} / \mathrm{mol}$ to $600 \times 10^{3} \mathrm{~g} / \mathrm{mol}$. The samples were dissolved in aqueous acetic acid, filtered through a Millipore membrane with reduced porosity (Millipore Sigma, Burlington, USA), precipitated with ammonia, rinsed to neutral with deionized water, and lyophilized. The purified high molar mass CS was acetylated with acetic anhydride in a homogeneous medium. The CS was dissolved at $0.5 \%(\mathrm{w} / \mathrm{v})$ in $0.2 \mathrm{M}$ acetic acid with $0.1 \mathrm{M}$ sodium acetate buffer. We added $0.15 \mathrm{M}$ sodium nitrite solution to the CS solution to obtain nitrite/glucosamine at a molar ratio of 0.5 . The reaction was carried out for various durations (15-60 min) under moderate magnetic stirring according to the target $\mathrm{Mw}$. The CS was recovered through ammonia precipitation, washed several times with deionized water until neutral and finally freeze-dried.

Hyaluronic acid with varying $\mathrm{Mw}$, ranging from $1500 \times 10^{3} \mathrm{~g} / \mathrm{mol}$ to $50 \times 10^{3} \mathrm{~g} / \mathrm{mol}$, was provided by Zhenjiang Dongyuan Biotechnology Co., Ltd. (Jiangsu, China). Anti-CD47 (CD47) antibody was provided by Santa Cruz Biotechnology (Santa Cruz, USA) and the concentrations were confirmed using a Pierce bicinchoninic acid (BCA) assay according to the manufacturer's instructions (Thermo Fisher Scientific, Hudson, USA).

\section{Preparation of polyelectrolyte solution}

The CS was dispersed in water or phosphate-buffered saline (PBS) at a concentration of $0.1 \%$ by mass. The solution was adjusted to $\mathrm{pH}=4.0$ with $0.1 \mathrm{M}$ sodium hydroxide or hydrochloric acid. All the CS solutions were filtered through a $0.22-\mu \mathrm{m}$ pore size Millipore membrane prior to use. A $0.1 \%$ strength HA solution was prepared directly in water or PBS medium with magnetic stirring.

\section{Formation of polyelectrolyte complexes}

Under non-stoichiometric conditions $(\mathrm{R}=\mathrm{n}+\mathrm{n}-\geq 1$, where $\mathrm{R}$ is the mixed molar charge ratio), excess polymer was added to the magnetic stirrer in 1 portion. A colloidal PEC (750 rpm) was formed with magnetic stirring at room temperature. The final particle dispersion volume was $30 \mathrm{~mL}$ and the solid content was $0.1 \%$. The substrate was precipitated from the solution by centrifugation 
at $7000 \mathrm{rpm}$ and $20^{\circ} \mathrm{C}$ for $10 \mathrm{~min}$. The supernatant was removed and the pellet was resuspended in deionized water or PBS. In all the experiments performed in this study, the initial solution of $\mathrm{CS}$ was adjusted to $\mathrm{pH}=4.0$ to ensure full protonation. Chitosan with an Mw of $100 \times 103 \mathrm{~g} / \mathrm{mol}$ and DA of $5 \%$ as well as with an Mw of $200 \times 103 \mathrm{~g} / \mathrm{mol}$ and DA of $48 \%$ was used. The average molar mass of the HA used ranged from $50 \times 103 \mathrm{~g} / \mathrm{mol}$ to $1500 \times 103 \mathrm{~g} / \mathrm{mol}$. Hyaluronic acid was complexed to $\mathrm{CS}$ and the charge ratio of the complex was controlled to be $\left(\mathrm{N}+/ \mathrm{N}_{-}\right)=1.5$.

\section{Determination of particle solid content}

The solid content is defined as the ratio between the weight of the particles after drying at $60^{\circ} \mathrm{C}$ for $24 \mathrm{~h}$ and the initial weight of the solution.

\section{Physical and chemical characterization of complex dispersions}

Dynamic light scattering measurements of the PEC dispersion were performed using a Malvern Nanosizer SZ (Malvern Instruments, Malvern, UK) at the Huazhong University of Science and Technology Analytical Testing Center (Wuhan, China) with a parameter of $\lambda=633 \mathrm{~nm}$ (at a $173^{\circ}$ scattering angle), operated using a $10 \mathrm{~mW} \mathrm{He} / \mathrm{Ne}$ laser beam. All the dynamic light scattering measurements were performed at least 3 times at $25^{\circ} \mathrm{C}$. The polydispersity index (PI) is derived from an algorithm provided by the software and is defined as $\mu 2 /(\Gamma) 2$, where $\mu 2$ is the second cumulative amount of the correlation function and $(\Gamma)$ is the average decay rate. Each polydispersity index measurement is the average of 6 measurements in 3 experiments. The zeta potential was measured using the electrophoretic mobility $(\mu \mathrm{E})$ from the Smoluchowski equation. The $\mu \mathrm{E}$ of the particles was measured at $25^{\circ} \mathrm{C}$ using the Malvern Nanosizer $\mathrm{SZ}$, and the measurements were carried out by suspending the washed dispersion in a $10^{-3} \mathrm{M}$ sodium chloride solution. The average of 10 measurements was used with a relative error of 5\%. A scanning electron microscope (SEM) image was obtained at $5 \mathrm{kV}$ on a Hitachi S-4800 microscope (Hitachi Ltd., Tokyo, Japan). A 0.01\% (v/v) nanoparticle dispersion droplet was deposited on a sample holder, air dried at room temperature for $12 \mathrm{~h}$ and coated under an argon atmosphere with a palladium cathode evaporator (Structure Probe; Inc./SPI Supplies, West Chester, USA). Scanning electron microscope examinations were performed at the Center for Analysis and Testing Technology of Huazhong University of Science and Technology.

\section{CD47 antibody adsorbed onto CS-HA polyelectrolyte nanocolloid}

Chitosan-hyaluronic acid polyelectrolytic nanocolloid (CS Mw $200 \times 10^{3} \mathrm{~g} / \mathrm{mol}$ and $\mathrm{DA}=48 \%$, HA $\left.\mathrm{Mw}=50 \times 10^{3} \mathrm{~g} / \mathrm{mol}\right)$ was mixed with different concentrations of the CD47 antibody solution with moderate agitation, so that the final mixed molar charge ratio of the mixture was $R=1.5$. After the mixture was centrifuged at $14,000 \mathrm{~g}$ for $10 \mathrm{~min}$, the supernatant was removed and the pellet was resuspended in the same volume of buffer. The anti-CD47 adsorption yield was calculated as follows: anti-CD47 adsorption rate $=(($ anti-CD47 input - anti-CD47 residue)/anti-CD47 input) $\times 100 \%$, where anti-CD47 input is the concentration of anti-CD47 titrated in the original control sample solution for each independent experiment, and anti-CD47 residue refers to the concentration of anti-CD47 measured with titration in the supernatant.

\section{In vitro targeting of VECs by anti-CD47-CS-HA polyelectrochemical complex}

The anti-CD47 nanocarrier was labeled with fluorescein isothiocyanate (FITC) fluorescent secondary antibody in vitro. The VEC strain was routinely cultured. When the cells were grown to $80 \%$ confluency, the medium was changed to a serum-free medium and cultured for 3 days in the absence of serum medium. The cells were added to $50 \mu \mathrm{g} / \mathrm{mL}$ ox-LDL for $72 \mathrm{~h}$, and then inoculated into a 24-well cell culture plate, co-cultured with anti-CD47 nano adjuvant for $24 \mathrm{~h}$ and washed with PBS. The medium was exchanged and the suspension of the adsorbed nanocarriers was simultaneously counterstained with DAPI. The number and proportion of green fluorescent cells were observed under a fluorescence microscope.

\section{Targeted experiment with synthetic anti-CD47-CS-HA polyelectrochemical complex for atherosclerotic plaque}

Atherosclerosis-prone apolipoprotein E-deficient (ApoE -/-) mice display poor lipoprotein clearance with subsequent accumulation of cholesterol ester-enriched particles in the blood, which promote the development of atherosclerotic plaque. Therefore, the ApoE -/- mouse model is well established for the study of atherosclerosis. A total of 24 ApoE mice were purchased from the Experimental Animal Center of Nanjing University (China). The mice were fed a high-fat and high-cholesterol diet, and were fostered in the Specific Pathogen-Free (SPF) animal room at the Experimental Animal Center of Tongji Medical College at Huazhong University of Science and Technology (Wuhan, China). After 20 weeks of feeding, the model of atherosclerosis was ready. For the last 8 weeks of the 20-week period, the animals were divided into 3 groups: the P group ( $\mathrm{n}=8)$, which was administered Cy5.5anti-CD47-CS-HA-PECs; the M group ( $\mathrm{n}=8$ ), given cy5.5 dye solution; and the G group $(\mathrm{n}=8)$, which got CS-HAPECs. Each mouse was injected with $0.2 \mathrm{~mL}$ of its assigned component in the tail vein once a week for 8 weeks. After 
the injections were completed in the $20^{\text {th }}$ week, the health status and blood cell count of each mouse were checked. Normal mice were used for follow-up experiments.

\section{In vivo targeting of atherosclerotic plaque in ApoE mice by Cy5.5-CD47 antibody-CS-HA-PECs}

Each group of mice began fasting $6 \mathrm{~h}$ before imaging to reduce potential background interference caused by gastrointestinal foods. Group $\mathrm{G}$ was injected with $0.2 \mathrm{~mL}$ PBS through the tail to dissolve $10 \mathrm{mg} / \mathrm{mL}$ CS-HA-PECs, whereas group P was injected with $0.2 \mathrm{~mL}$ PBS to dissolve Cy5.5-labeled (25 ug) anti-CD47-CS-HA-PECs (10 mg/mL). The control group $(\mathrm{M})$ was injected with $0.1 \mathrm{~mL}$ of a free $\mathrm{Cy} 5.5$ dye solution $(1 \mathrm{mg} / \mathrm{mL})$ through the tail vein. Twenty four hours after the injection, the mice were anesthetized with an intraperitoneal injection of $10 \%$ chloral hydrate solution $(0.35 \mathrm{~mL}$ per $100 \mathrm{mg}$ of body weight). After anesthesia, the mice were depilated to reduce the background fluorescence interference generated by the hair and fixed onto the treatment bed with tape in a supine position. The small animal in vivo imaging system IVIS 200 (Perkin Elmer, Waltham, USA) was used to image the entire body; the thorax was imaged with the fluorescence scanning in vivo imaging system of the same device. The images of the animals emitting fluorescence in vivo were recorded and the distribution of the fluorescent complex Cy5.5-CD47 antibody-CS-HA-PECs was analyzed. The fluorescence distribution of the negative and positive control groups was compared. After the imaging, the aortas were harvested and tissue sections were prepared. The limbs of the mice were fixed in an anatomical tray, and the chest and abdomen were smeared with $75 \%$ alcohol until the skin was moist. The abdominal skin was lifted with a sterilized shaver and surgically cut with scissors. A gap was carefully cut to open the chest and abdominal cavity and expose the heart. Physiological saline was used to perfuse the heart followed by an infusion of $10 \%$ formaldehyde for about $40 \mathrm{~min}$. The blood vessel and surrounding connective tissue were removed from the root of the aorta, and the abdominal aorta was harvested. Several 1-1.5-cm specimens were cut and fixed with $10 \%$ formaldehyde. After $48 \mathrm{~h}$, the tissues were embedded in paraffin and $0.2-\mathrm{cm}$ samples with a thickness of $5 \mu \mathrm{m}$ were evenly sliced from the aortic root. Five sections per blood vessel were observed with fluorescence microscopy.

\section{Statistical analysis}

All data was expressed as means \pm standard deviation (SD), and the statistical analyses were performed using IBM SPSS Statistics for Windows v. 20.0 (IBM Corp., Armonk, USA). Comparisons among groups were analyzed using a one-way analysis of variance (ANOVA), followed by the Student-Newman-Keuls test. A p $<0.05$ was considered statistically significant.

\section{Results and discussion}

\section{Formation of polyelectrolyte complexes}

Chitosan interacts with the ionized carboxyl group (COO-) between ionized aminoglycan (NH3+) and HA to form PECs (Fig. 1). The various colloidal dispersions obtained in the study were measured to determine their average size and the particle size distribution after complexation. An increase in the molar mass of HA resulted in a significant increase in the particle size (Fig. 2; the data represented the mean \pm SD of 3 independent tests; " $\mathrm{p}<0.05)$. The $1500 \times 10^{3} \mathrm{~g} / \mathrm{mol} \mathrm{HA}$ sample did not form particles in the experiment; only a precipitate was obtained. This indicates that such a high Mw polymer cannot be unraveled to form a single particle, and only aggregated precipitates can be formed. Therefore, further experiments were not performed with $\mathrm{HA}$ at this molar mass. According to the trend shown in Fig. 2, as the molar mass of HA increases, the particle diameter of the resulting polyelectrolyte increases and the dynamic light scattering PI of the dispersion also increases. Therefore, the optimum molar mass for sodium hyaluronate - i.e., the smallest particle size and PI of the particles - was $50 \times 10^{3} \mathrm{~g} / \mathrm{mol}$.

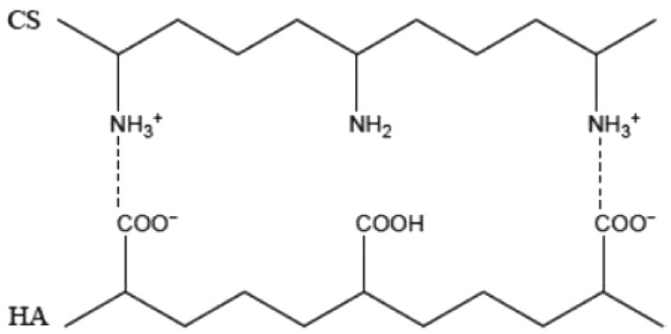

Fig. 1. Schematic diagram of a polyelectrolyte complexation reaction between $\mathrm{CS}$ and $\mathrm{HA}^{24}$

As shown in Fig. 3, increasing the molar mass of CS increased the average particle size of the polyelectrochemical composite PECs regardless of the acetylation rate, and the PI followed the same trend. These results suggest that an increase in the molar mass of CS will increase the size of CS-HA polyelectrolyte nanoparticles (data taken from the mean of 3 independent tests $\pm S D, p<0.05)$. The results show that there is a critical molar mass for CS. Above this point, the diameter of the polyelectro-composite nanoparticles will increase sharply. The critical molar mass is $200 \times 10^{3} \mathrm{~g} / \mathrm{mol}$ when the acetylation rate is $5 \%$ and $48 \%$. When the molar mass of CS is lower than this value, the granular structure formed is dense and uniform.

Therefore, to obtain small and fairly uniformly dispersed particles, low to medium molar masses of CS (about $200 \times 10^{3} \mathrm{~g} / \mathrm{mol}$ or less) and $50 \times 10^{3} \mathrm{~g} / \mathrm{mol} \mathrm{HA}$ are required. The results also show that the CS acetylation rate has a limited effect on the formation of polyelectro-composite nanoparticles. 

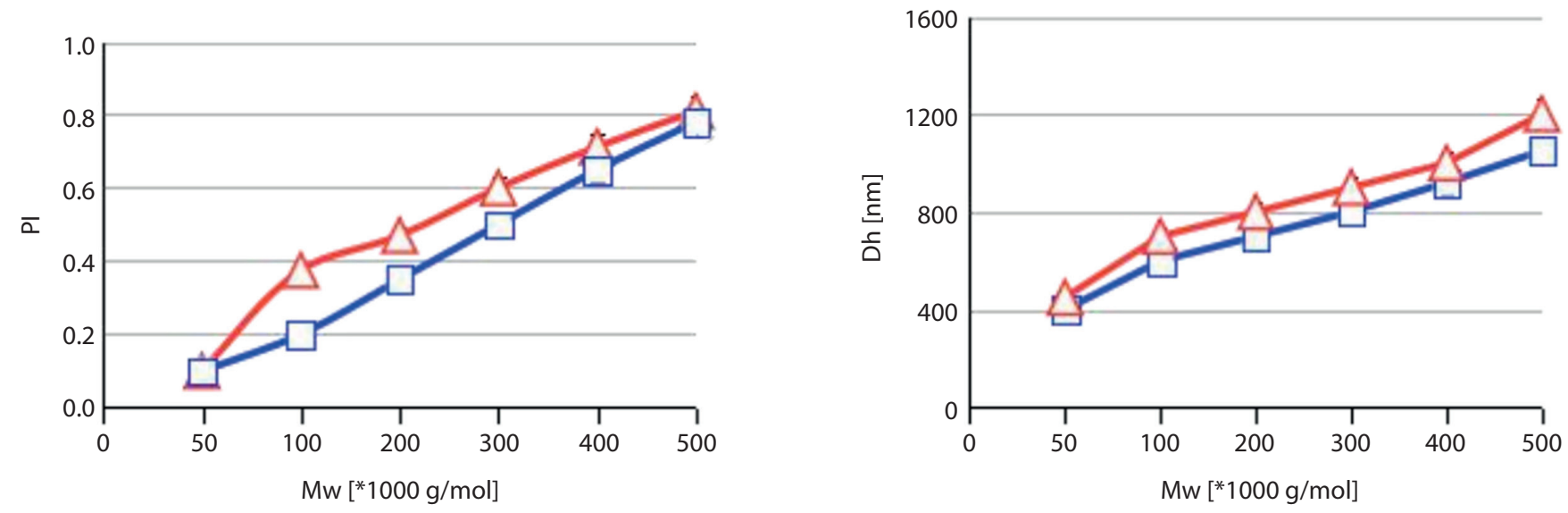

Fig. 2. The effect of HA with different Mw on the size and dispersion index (PI) of PECs was studied. The complex reacted with CS with a Mw of $10 \times 10^{3} \mathrm{~g} / \mathrm{mol}$ and a degree of acetylation (DA) of $5 \%(\Delta)$, as well as with CS with a Mw of $200 \times 10^{3} \mathrm{~g} / \mathrm{mol}$ and a DA rate of $48 \%$ ( $\mathbf{}$ ), with a charge ratio $\mathrm{R}$ of 1.5 in water. The data represents the average value and SD of 3 independent experiments $(p<0.05)$
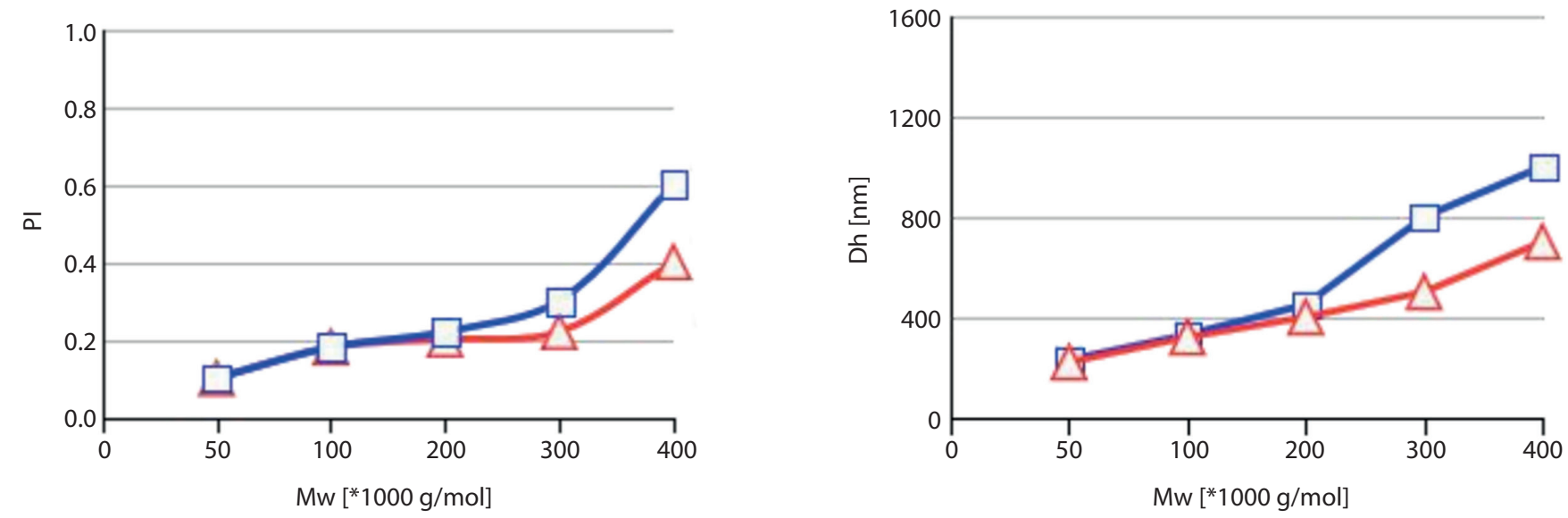

Fig. 3. Particle size and Mw distribution (PI) of polyelectrolyte complexes (PECs) affected by the Mw of CS. Chitosan DA was $5 \%$ ( $\Delta$ ) and $48 \%$ ( $\square$ ). Chitosan with Mw ranging from $20 \times 10^{3} \mathrm{~g} / \mathrm{mol}$ to $500 \times 10^{3} \mathrm{~g} / \mathrm{mol}$ and $\mathrm{HA}$ with a molar mass of $50 \times 10^{3} \mathrm{~g} / \mathrm{mol}$ reacted in water at a charge-to-charge ratio of $\mathrm{R}=1.5$. The data represents the average value and SD of 3 independent experiments $(p<0.05)$

\section{The effect of charge change on the physical and chemical properties of the formed polyelectrochemical complex}

As described above, the anionic and cationic polyelectrolyte in the mixed solution form a sub-electrolyte complex, and in both extreme cases, a completely water-soluble complex or precipitate is obtained. In order to obtain PEC nanocolloids that do not accumulate over time, the experiments in this study were focused on the effect of charge changes on the stability of the formed polyelectrochemical complex. The DA of CS was $48 \%$, the molar mass was $200 \times 10^{3} \mathrm{~g} / \mathrm{mol}$, and the low molar mass of HA was $50 \times 10^{3} \mathrm{~g} / \mathrm{mol}$ in water or PBS with a charge ratio $\mathrm{R}(\mathrm{N}+/ \mathrm{N}-)$ : $0.5,1.5,2.5$, and 3.5. Polyelectrolyte nanoparticles were obtained in water and PBS. Table 1 lists the size, PI, zeta potential and appearance of these nanoparticles (Table 1).

When $\mathrm{R}(\mathrm{N}+/ \mathrm{N}-)=0.5$, flocculation was observed with naked eye, as well as after reconstitution $24 \mathrm{~h}$ later. This may be due to the rearrangement of the initially formed complex and thus the observed re-dissolution. More HA participates in the reaction during polymerization, resulting in the redistribution of neutralized fragments, thereby forming a soluble complex. When the charge ratio is close to 1 , the size of the particles is significantly increased due to the complete neutralization of the charge. Due to the neutral, non-charged environment, there is no electrostatic charge to counteract the van der Waals interactions, leading to precipitation of the particles. When the charge ratio is increased from 1 , the particle size is reduced, and the colloidal stability is enhanced due to an increase in the amount of net charge on the particles. When $\mathrm{R}\left(\mathrm{N}_{+} / \mathrm{N}_{-}\right)$is from 1.5 to 3.5 , the polyelectrolyte nanoparticles have a diameter distribution between $230 \mathrm{~nm}$ and $320 \mathrm{~nm}$ in water and a diameter of $330-590 \mathrm{~nm}$ in PBS. The zeta potential of the polyelectrochemical complex is positive. As the CS incorporated into the colloid increases, the charge ratio $\mathrm{R}$ increases and so did the zeta potential, 
Table 1. Physicochemical characteristics of colloidal PECs after synthesis at different charge ratios: CS (DA 48\%, Mw $200 \times 10^{3} \mathrm{~g} / \mathrm{mol}$ ) and HA $\left(\mathrm{Mw} 50 \times 10^{3} \mathrm{~g} / \mathrm{mol}\right)$

\begin{tabular}{|c|c|c|c|c|c|c|}
\hline $\mathrm{CS}-\mathrm{HA}$ & $\begin{array}{c}\text { Charge ratio } \\
(n+/ n-)\end{array}$ & Solid content (\%) & Size $[n m]$ & $\mathrm{PI}$ & Zeta potential [mV] & Appearance \\
\hline \multirow{4}{*}{ Water } & 0.5 & - & 1050 & 1 & - & precipitation \\
\hline & 1.5 & 5 & 320 & 0.1 & $25 \pm 1.63$ & medium turbidity \\
\hline & 2.5 & 2 & 260 & 0.1 & $31 \pm 0.23$ & medium turbidity \\
\hline & 3.5 & 1 & 230 & 0.1 & $43 \pm 0.17$ & low turbidity \\
\hline \multirow{4}{*}{ PBS } & 0.5 & - & 1180 & 1 & - & precipitation \\
\hline & 1.5 & 4 & 590 & 0.1 & $32 \pm 0.13$ & medium turbidity \\
\hline & 2.5 & 3 & 392 & 0.1 & $42 \pm 0.27$ & medium turbidity \\
\hline & 3.5 & 1 & 330 & 0.1 & $45 \pm 0.31$ & low turbidity \\
\hline
\end{tabular}

PEC - polyelectrolyte complex; HA - hyaluronic acid; CS - chitosan; PBS - phosphate-buffered saline; PI - polydispersity index; DA - degree of acetylation; Mw - molecular weight.

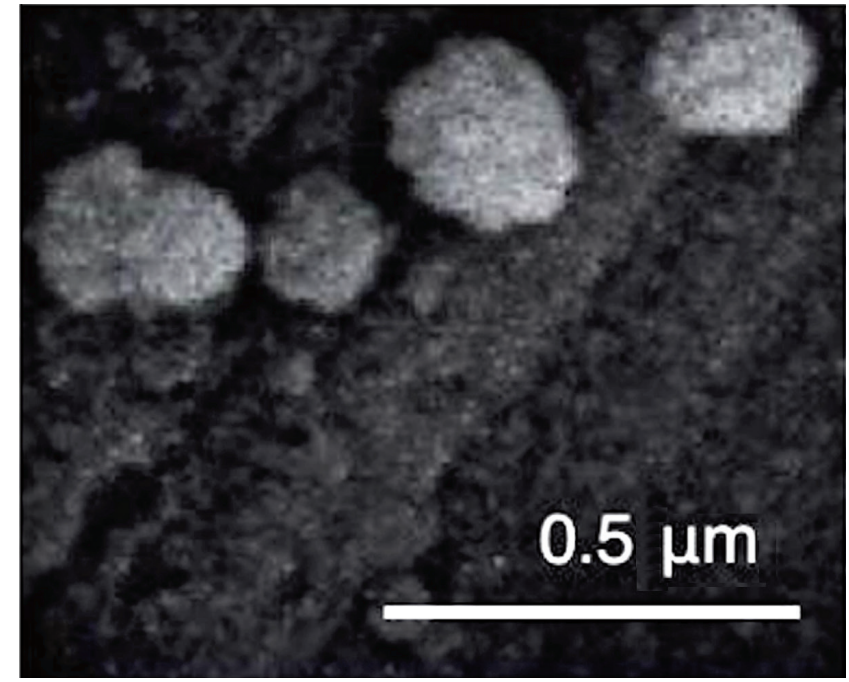

Fig. 4. Scanning electron microscopy images showing the ultrastructure of the CS-HA polyelectric complex (CS: DA 48\%, molar mass $200 \times 10^{3} \mathrm{~g} / \mathrm{mol} ; \mathrm{HA}: 500 \times 10^{3} \mathrm{~g} / \mathrm{mol}$, charge ratio $\left.\mathrm{R}=1.5: 1\right)$

as expected. Scanning electron microscopy was used to detect the spherical morphology of CS-HA nanoparticles. Figure 4 shows the ultrastructure of the CS-HA $\mathrm{PEC}$ composite that had $\mathrm{R}=1.5$, and the particles clearly show a rough surface.

\section{Colloidal stability}

The average size of the particles was measured with elastic light scattering. Most samples in aqueous solution are stable for at least 1 month. When $\mathrm{R}=1.5$, the dispersion index PI value increased significantly on the $30^{\text {th }}$ day of observation (Tables 2, 3).

The colloidal particles of the polyelectrochemical composite PECs have a smaller particle size in water than in PBS, reflecting the electrostatic stability mode of the PEC composite. Due to the hydrophobic action, the chain inside the CS extends to the hydrophilic peripheral region, thereby preventing aggregation of the polymer. Due to the electrostatic action of salt ions in the PBS solution, the stabilization process for the PEC polymer is not as effective as in water, and thus the particles have a larger average diameter. As shown in Table 3, we observed 2 significant characteristics of the change in particle diameter: First, as the charge ratio $\mathrm{R}$ increased, the diameter of the particles became smaller. Second, regardless of the type of solution and the ratio of charge, the diameter of the particle decreased with time. This may be due to the reorganization of the particle structure during the process. Nonetheless, after storage for 1 month at room temperature, the particle size distribution PI index of the solution increased, indicating that the dispersion range of the particles had expanded.

Table 3. CS-HA nanoparticle colloidal stability with time as a function of charge ratio in PBS (CS: DA $48 \%$, molar mass $200 \times 10^{3} \mathrm{~g} / \mathrm{mol}$; $\left.\mathrm{HA}: 50 \times 10^{3} \mathrm{~g} / \mathrm{mol}\right)$

\begin{tabular}{|c|c|c|c|c|c|c|}
\hline \multirow{3}{*}{ Time [days] } & \multicolumn{6}{|c|}{$\mathrm{CS}-\mathrm{HA}$ in PBS } \\
\hline & \multicolumn{2}{|c|}{ Charge ratio $(n+/ n-)=1.5$} & \multicolumn{2}{|c|}{ Charge ratio $(\mathrm{n}+/ \mathrm{n}-)=2.5$} & \multicolumn{2}{|c|}{ Charge ratio $(n+/ n-)=3.5$} \\
\hline & size $[\mathrm{nm}]$ & $\mathrm{PI}$ & size $[\mathrm{nm}]$ & $\mathrm{PI}$ & size [nm] & $\mathrm{PI}$ \\
\hline 0 & 603 & 0.1 & 404 & 0.2 & 398 & 0.1 \\
\hline 7 & 591 & 0.1 & 381 & 0.2 & 365 & 0.1 \\
\hline 14 & 553 & 0.1 & 372 & 0.2 & 320 & 0.1 \\
\hline 30 & 306 & 0.3 & 225 & 0.2 & 216 & 0.2 \\
\hline
\end{tabular}

HA - hyaluronic acid; CS - chitosan; PBS - phosphate-buffered saline; PI - polydispersity index; DA - degree of acetylation; Mw - molecular weight. 
Table 2. CS-HA nanoparticle colloidal stability with time as a function of charge ratio in water (CS: DA $48 \%, \mathrm{molar}$ mass $200 \times 10^{3} \mathrm{~g} / \mathrm{mol}$; $\mathrm{HA}: 50 \times 10^{3} \mathrm{~g} / \mathrm{mol}$ )

\begin{tabular}{|c|c|c|c|c|c|c|}
\hline \multirow{3}{*}{ Time [days] } & \multicolumn{6}{|c|}{ CS-HA in water } \\
\hline & \multicolumn{2}{|c|}{ Charge ratio $(n+/ n-)=1.5$} & \multicolumn{2}{|c|}{ Charge ratio $(n+/ n-)=2.5$} & \multicolumn{2}{|c|}{ Charge ratio $(\mathrm{n}+/ \mathrm{n}-)=3.5$} \\
\hline & size $[\mathrm{nm}]$ & $\mathrm{PI}$ & size $[\mathrm{nm}]$ & $\mathrm{PI}$ & size $[\mathrm{nm}]$ & $\mathrm{PI}$ \\
\hline 0 & 320 & 0.1 & 280 & 0.1 & 260 & 0.1 \\
\hline 7 & 300 & 0.1 & 242 & 0.1 & 253 & 0.1 \\
\hline 14 & 250 & 0.2 & 223 & 0.1 & 203 & 0.1 \\
\hline 30 & 152 & 0.3 & 201 & 0.2 & 121 & 0.2 \\
\hline
\end{tabular}

HA - hyaluronic acid; CS - chitosan; PI - polydispersity index; DA - degree of acetylation; Mw - molecular weight.

In vitro targeted adsorption of VECS by synthetic anti-CD47-CS-HA nanopolymerization complex

The DAPI staining highlighted the nuclei of all VECs, which appeared green under the fluorescence microscope, showing anti-CD47-targeting nanoparticles adsorbed onto the VECs. The results demonstrated that anti-CD47-targeting nanocarriers can effectively adsorb to VECs. On the surface, the effective adsorption rate was about $90 \%$, and the formula for the effective adsorption rate $=$ the number of green fluorescent positive cells/ the number of DAPI blue fluorescent positive cells (Fig. 5).

\section{Synthesis of anti-CD47-CS-HA nanopolymer complex for targeted adsorption of atherosclerotic plaque}

The results showed that compared with the control group, the fluorescently labeled CD47 antibody-CS-HA-PECs
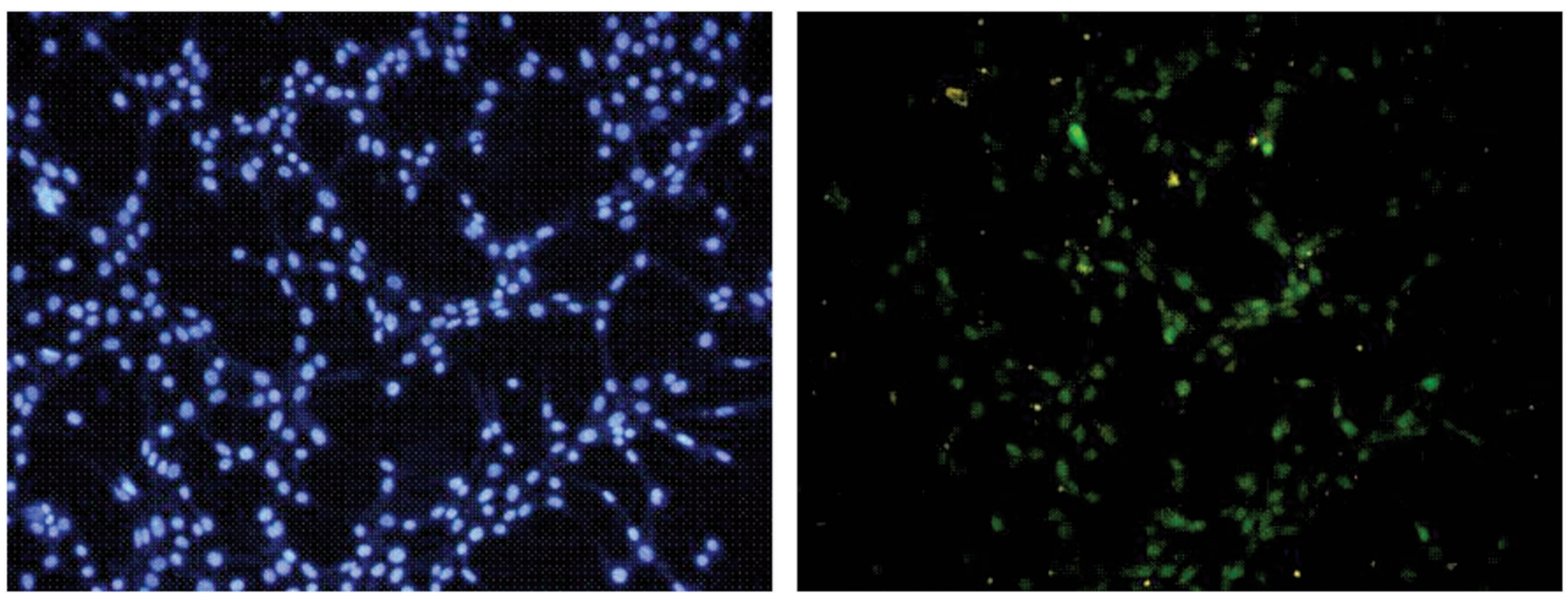

Fig. 5. The interaction of FITC-labeled anti-CD47-targeting CS-HA nanoelectro complex with cultured VEC lines in vitro

A - DAPI staining showed the nuclei of all VECS; B - FITC fluorescence microscopy showed that anti-CD47-targeting nanoparticles were absorbed on VECs. The experimental results showed that anti-CD47-targeting nanocarriers could be effectively absorbed to the surface of vascular endothelium.

Table 4. CD47 sorption yield from protein solutions in water or PBS at different concentrations. CS-HA particles at a ratio R $=1.5\left(\mathrm{CS}: \mathrm{Mw} 200 \times 10^{3} \mathrm{~g} / \mathrm{mol}\right.$ and $\left.\mathrm{DA}=48 \% ; \mathrm{HA}: \mathrm{Mw}=50 \times 10^{3} \mathrm{~g} / \mathrm{mol}\right)$

\begin{tabular}{|c|c|c|c|c|c|c|}
\hline \multirow{3}{*}{ Time [h] } & \multicolumn{6}{|c|}{ Adsorption rate (\%) } \\
\hline & \multicolumn{3}{|c|}{ input of CD47 in PBS [g/L] } & \multicolumn{3}{|c|}{ input of CD47 in water [g/L] } \\
\hline & 0.1 & 0.3 & 0.5 & 0.2 & 0.4 & 0.6 \\
\hline 2 & 95 & 91 & 90 & 93 & 91 & 85 \\
\hline 4 & 97 & 98 & 95 & 95 & 93 & 90 \\
\hline 6 & 100 & 99 & 97 & 99 & 99 & 95 \\
\hline 16 & 100 & 100 & 100 & 100 & 100 & 100 \\
\hline 24 & 100 & 100 & 100 & 100 & 100 & 100 \\
\hline
\end{tabular}

PEC - polyelectrolyte complex; HA - hyaluronic acid; CS - chitosan; PBS - phosphate-buffered saline; PI - polydispersity index; DA - degree of acetylation; $\mathrm{Mw}$ - molecular weight. 


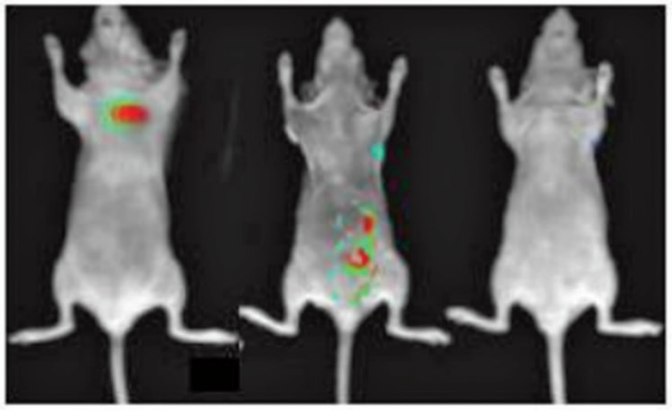

P group M group G group

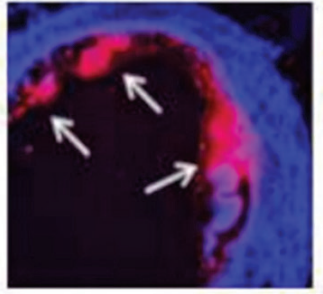

P group

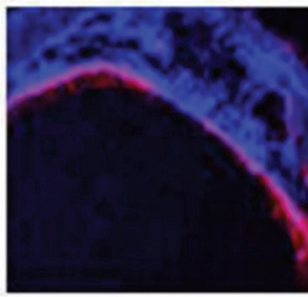

M group

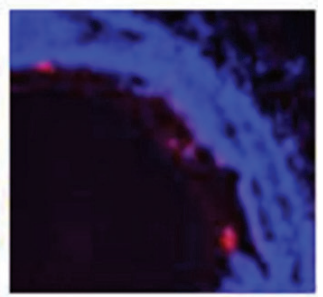

G group

P group: Cy5.5-CD47-CS-HA-PECS

M group: Cy5.5 dye solution

G group: CS-HA-PECS

Fig. 6. Targeted adsorption of anti-CD47-CS-HA nanocomposite on atherosclerotic plaque. Using the IVIS 200 in vivo imaging system for small animals, images of fluorescence emission from animals were recorded and the Cy5.5-CD47 antibody-CS-HA-PECs were distributed around atherosclerotic plaques in vivo and compared with the control group. No fluorescence was detected in group G mice, which lacked antibody targeting labeling. The fluorescence distribution in group M labeled with fluorescent Cy5.5 dye solution alone is shown (left). After imaging, the aorta was harvested and sliced into sections. Five sections were taken from each vessel and the nuclei were stained with DAPI. Fluorescence microscopy confirmed the targeting of CD47 antibody-CSHA-PECS to atherosclerotic plaque in vivo (right)

were distributed around the atherosclerotic plaque in vivo. Group G, without the antibody-targeted marker, showed no fluorescence in the body. The fluorescence distribution of the control group (M) labeled with the Cy5.5 dye solution was not localized to the plaque (Fig. 6). The fluorescence of the CD47 antibody-CS-HA-PECs was confirmed in vivo to target atherosclerotic plaque (Fig. 6).

\section{Conclusions}

In this work, experiments were conducted to obtain positively charged PEC nanoparticles from HA and CS which maintained their colloidal stability in physiological media for 30 days at room temperature. Antibodies can be quantitatively adsorbed at the particle interface without changing colloidal stability.

With reference to the relevant literature and our preliminary results, the following parameters were selected: low and medium molar mass CS (about $200 \times 10^{3} \mathrm{~g} / \mathrm{mol}$ and lower), HA with a molar mass of about $50 \times 10^{3} \mathrm{~g} / \mathrm{mol}$ and a charge ratio $\mathrm{R}(\mathrm{n}+/ \mathrm{n}-)$ maintained in the range from 1.5 to 3.5. The experiments were carried out in deionized water or PBS and the pellet solid content was increased to $5 \%$ after centrifugation and re-dispersion. The diameter of the resulting nanoparticles decreased as R increased, but the conversion of the polymer to a colloid decreased. The results showed that the acetylation ratio of CS has little effect on the particle formation process.

The association of anti-CD47 with CS-HA nanoparticles resulted in rapid kinetics and high load capacity in both water and PBS. After anti-CD47 loading, the particle sizes observed in water and PBS were $375 \mathrm{~nm}$ and $620 \mathrm{~nm}$, respectively, and the zeta potential remained positive. The CS-HA/anti-CD47 polyelectron nanoparticles remained stable for 3 weeks in both buffers. These results indicate that CS-HA polyelectron nanoparticles have a high loading capacity for CD47 antibody and maintain good colloidal stability in PBS. More importantly, anti-CD47 nanocarriers were labeled with FITC fluorescent secondary antibody in vitro. The VEC strain was inoculated into a 24-well cell culture plate and co-cultured with CD47 nano-adjuvant for $24 \mathrm{~h}$. The results showed that the anti-CD47-targeted nanocarrier can effectively adsorb to VECs. On the surface, the effective adsorption rate was about $90 \%$ and the nanoparticles targeted atherosclerotic plaques in in vivo experiments. Therefore, both CS and HA are non-toxic polysaccharides derived from biomass, laying the foundation for further research on the use of CD47 antibodies to target functional biopharmaceutical delivery nanosystems against atherosclerosis.

\section{ORCID iDs}

Jun Yu (D) https://orcid.org/0000-0002-3390-5901 Qiurong Ruan (D) https://orcid.org/0000-0002-7015-7666 Xiu Nie (D) https://orcid.org/0000-0002-1001-7709

\section{References}

1. Kojima Y, Volkmer JP, McKenna K, et al. CD47-blocking antibodies restore phagocytosis and prevent atherosclerosis. Nature. 2016; 536(7614):86-90. doi:10.1038/nature18935

2. Lankalapalli S, Kolapalli VRM. Polyelectrolyte complexes: A review of their applicability in drug delivery technology. Indian J Pharm Sci. 2009;71(5):481. doi:10.4103/0250-474x.58165

3. Sun W, Mao S, Mei D, Kissel T. Self-assembled polyelectrolyte nanocomplexes between chitosan derivatives and enoxaparin. Eur JPharm Biopharm. 2008;69(2):417-425. doi:10.1016/j.ejpb.2008.01.016

4. Vaidyanathan K, Gopalakrishnan S. Nanomedicine in the diagnosis and treatment of atherosclerosis: A systematic review. Cardiovasc Hematol Disord Drug Targets. 2017;17(2):119-131. doi:10.2174/187152 9x17666170918142653

5. Vila A, Sánchez A, Tobío M, Calvo P, Alonso M. Design of biodegradable particles for protein delivery. J Control Release. 2002;78(1):15-24. doi:10.1016/s0168-3659(01)00486-2

6. Rodrigues FC, Singh K, Thakur G. Pharmaceutical application of chitosan derivatives. In: Nayak AK, Hasnain S, Pal D, eds. Natural Polymers for Pharmaceutical Applications. Vol. 2. New York, NY: Apple Academic Press; 2019:141-163. doi:10.1201/9780429328299-6 
7. Kean T, Thanou M. Biodegradation, biodistribution and toxicity of chitosan. Adv Drug Deliv Rev. 2010;62(1):3-11. doi:10.1016/j.addr. 2009.09.004

8. Kim SJ, Yoon SG, Lee KB, Park YD, Kim SI. Electrical sensitive behavior of a polyelectrolyte complex composed of chitosan/hyaluronic acid. Solid State lonics. 2003;164(3):199-204. doi:10.1016/j.ssi.2003.08.005

9. Chen WB, Wang LF, Chen JS, Fan SY. Characterization of polyelectrolyte complexes between chondroitin sulfate and chitosan in the solid state. J Biomed Mater Res A. 2005;75(1):128-137. doi:10.1002/ jbm.a.30393

10. Komoto D, Furuike T, Tamura H. Preparation of polyelectrolyte complex gel of sodium alginate with chitosan using basic solution of chitosan Int J Biol Macromol. 2019;126:54-59. doi:10.1016/j.ijbiomac.2018.12.195

11. Karzar Jeddi M, Mahkam M. Magnetic nano carboxymethyl cellulose-alginate/chitosan hydrogel beads as biodegradable devices for controlled drug delivery. Int J Biol Macromol. 2019;135:829-838. doi:10.1016/j.ijbiomac.2019.05.210

12. Volod'ko AV, Davydova VN, Nedashkovskaya Ol, et al. Morphology, electrokinetic characteristics and the effect on biofilm formation of carrageenan: Chitosan polyelectrolyte complexes. Int J Biol Macromol. 2018;117:1118-1124. doi:10.1016/j.ijbiomac.2018.05.215

13. Khanna R, Katti KS, Katti DR. Bone nodules on chitosan-polygalacturonic acid-hydroxyapatite nanocomposite films mimic hierarchy of natural bone. Acta Biomater. 2011;7(3):1173-1183. doi:10.1016/j. actbio.2010.10.028

14. Liu W, Sun S, Cao Z, et al. An investigation on the physicochemical properties of chitosan/DNA polyelectrolyte complexes. Biomaterials. 2005;26(15):2705-2711. doi:10.1016/j.biomaterials.2004.07.038

15. Prestwich GD. Hyaluronic acid-based clinical biomaterials derived for cell and molecule delivery in regenerative medicine. J Control Release. 2011;155(2):193-199. doi:10.1016/j.jconrel.2011.04.007

16. Yun YH, Goetz DJ, Yellen P, Chen W. Hyaluronan microspheres for sustained gene delivery and site-specific targeting. Biomaterials. 2004;25(1):147-157. doi:10.1016/s0142-9612(03)00467-8
17. Oh EJ, Park K, Kim KS, et al. Target specific and long-acting delivery of protein, peptide, and nucleotide therapeutics using hyaluronic acid derivatives. J Control Release. 2010;141(1):2-12. doi:10.1016/j. jconrel.2009.09.010

18. Yamane S, Iwasaki N, Majima T, et al. Feasibility of chitosan-based hyaluronic acid hybrid biomaterial for a novel scaffold in cartilage tissue engineering. Biomaterials. 2005;26(6):611-619. doi:10.1016/j. biomaterials.2004.03.013

19. Hahn SK, Hoffman AS. Preparation and characterization of biocompatible polyelectrolyte complex multilayer of hyaluronic acid and poly-L-lysine. Int J Biol Macromol. 2005;37(5):227-231. doi:10.1016/j. ijbiomac.2005.11.010

20. Malay O, Batigün A, Bayraktar O. pH- and electro-responsive characteristics of silk fibroin-hyaluronic acid polyelectrolyte complex membranes. Int J Pharm. 2009;380(1-2):120-126. doi:10.1016/j.ijpharm. 2009.07.011

21. Lu HD, Zhao HQ, Wang K, Lv LL. Novel hyaluronic acid-chitosan nanoparticles as non-viral gene delivery vectors targeting osteoarthritis. Int J Pharm. 2011;420(2):358-365. doi:10.1016/j.ijpharm.2011.08.046

22. Duceppe N, Tabrizian M. Factors influencing the transfection efficiency of ultra-low molecular weight chitosan/hyaluronic acid nanoparticles. Biomaterials. 2009;30(13):2625-2631. doi:10.1016/j.biomaterials. 2009.01.017

23. Verheul RJ, Slütter B, Bal SM, Bouwstra JA, Jiskoot W, Hennink WE. Covalently stabilized trimethyl chitosan-hyaluronic acid nanoparticles for nasal and intradermal vaccination. J Control Release. 2011; 156(1):46-52. doi:10.1016/j.jconrel.2011.07.014

24. Chua PH, Neoh KG, Kang ET, Wang W. Surface functionalization of titanium with hyaluronic acid/chitosan polyelectrolyte multilayers and RGD for promoting osteoblast functions and inhibiting bacterial adhesion. Biomaterials. 2008;29(10):1412-1421. doi:10.1016/j.biomaterials.2007.12.019 